\title{
Molecular model of thylakoid membrane bound (SlAPX6) ascorbate peroxidase from Solanum lycopersicum
}

\author{
Kaushlendra Tripathi1, 2, Saurabh Pandey1, Murtaza Malik1, 2, Krishnamurthy Sathelly, \\ Tanushri Kaul ${ }^{1}$
}

${ }^{1}$ Nutritional Crop Improvement Group, International Centre for Genetic Engineering and Biotechnology, Aruna Asaf Ali Marg, New Delhi-110067, India; 2Department of Biotechnology, Shri Venkateshwara University Venkateshwara Nagar, Rajabpur Gajraula, Amroha, Uttar Pradesh, 244236, India; Tanushri Kaul Email: tanushrikaul@gmail.com; tanushri@icgeb.res.in; Phone: +911126742962; +91- 9999966205: *Corresponding author

Received February 15, 2016; Revised March 02, 2016; Accepted March 03, 2016; Published April 10, 2016

\begin{abstract}
:
Ascorbate peroxidase (E 1.11.1.11) acts as primary key component of plant defense against photo protection and photo-oxidative stress. Chloroplastic (APX) located in the thylakoid membrane (tAPX) and stroma (sAPX) have been thought to be key regulators of intracellular levels of $\mathrm{H}_{2} \mathrm{O}_{2}$. Therefore, it is of interest to study thylakoid membrane bound SlAPX from Solanum lycopersicum (tomato, a fleshy fruit). However, a structure model is not yet solved for tomato thylakoid membrane bound SlAPX. "Hence, a homology molecular model of SlAPX6 from S. lycopersicum was constructed using a template structure (PDB ID: 1APX) from Pisum sativum. The model was further assessed using accessible surface area (ASA) calculations to identify surface residues for further characterization of active site regions. We further characterized the active site regions in the enzyme for functional inference. This information provides insights for the understanding of photo protection and photo-oxidative stress tolerant in S. lycopersicum during flower development and fruit ripening."
\end{abstract}

\section{Background:}

Tomato (S. lycopersicum) is fleshy fruit an important crop since of its great nutritive and commercial value of world, and also a good model plant for study fruit development with the release of the whole genome sequence of tomato. Now, it is very suitable to comprehensive structural and functional analysis of entire potential genes. Photooxidative stress is one of mainly important environmental factors that influence flower and fruit development stage (green, breaker and red). Photooxidative stress in the plant cell owing to higher reduced of electrons towards $\mathrm{O}_{2}$ during photosynthesis and respiratory processes leading to enhancement to generation of reactive oxygen species. ROS can directly attack inactivates metabolic enzymes and DNA damage and membrane lipids leading to programmed cell death [1]. APX (EC, 1.11.1.11) is known play essential role in scavenging $\mathrm{ROS}$ and protecting cells against plant stress and it catalyze the conversion of $\mathrm{H}_{2} \mathrm{O}_{2}$ to $\mathrm{H}_{2} \mathrm{O}$ using ascorbate as the specific electron donor [2]. In tomato, complete genome information of SIAPX gene isoforms is comprised of genes encoding cytosolic (SlAPX1, and SlAPX2), peroxisomal ISSN 0973-2063 (online) 0973-8894 (print)
(SlAPX3, and SlAPX4), and chloroplastic APX (SlAPX5, and $S I A P X 6)$. There is increasing interest in the chloroplastic thylakoid membrane bound SIAPX6 genes in tomato for enhancing tolerance of photooxidative stress [3]; however, there is lacking information on the protein structure of the tomato SIAPX6.

In this paper reports structural modeling of thylakoid membrane bound (SIAPX6) from tomato was selected for which three dimensional structures were neither available at the protein data bank (PDB) and no sufficient information available in structural level. Hence, we studied the protein structure model of SIAPX6 from tomato.

\section{Methodology:}

Template Selection and Protein sequence analysis:

The sequence of the tomato SIAPX6 (TbAPX) was retrieved from Sol Genomics Network https://solgenomics.net/search/ database with accession number Solyc11g018550.2.1. Template selection was done using BLASTp [4] for the query sequence 
against Protein Data Bank) database [5]. The target subsequently selected was the $x$ ray crystal structure with PDB code 1APX. Sequence alignments were completed employing ClustalW [6]. ScanProsite [7] was used to identify motif.

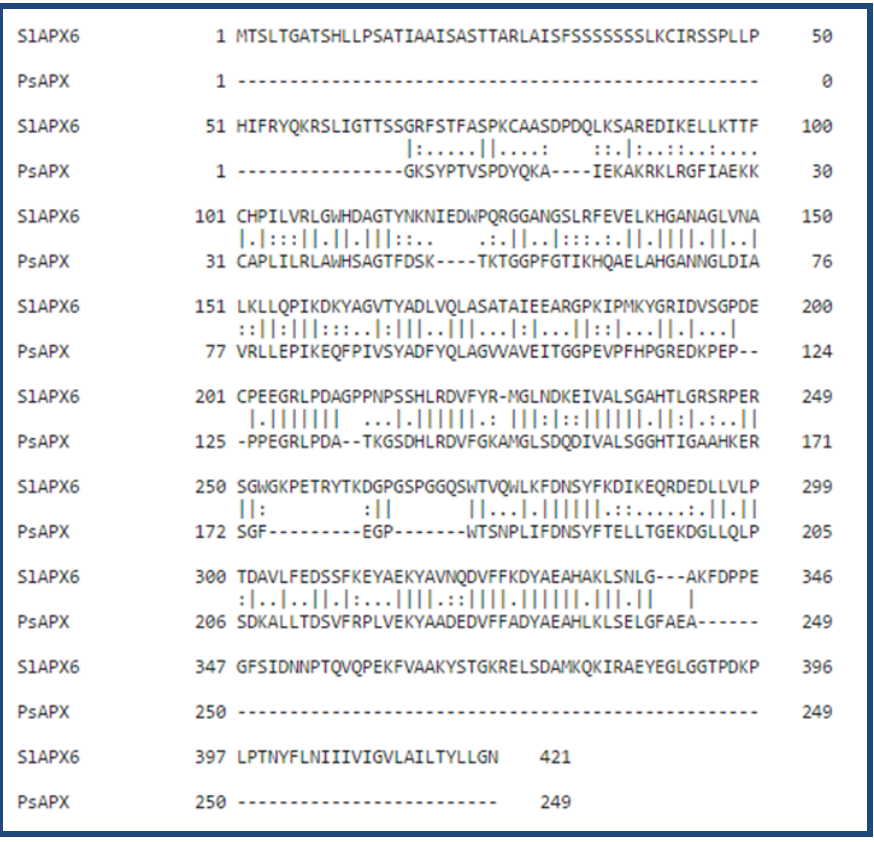

Figure 1: Sequences alignment SlAPX6 (target) and PsAPX (template) showing conserved patterns of protein sequences

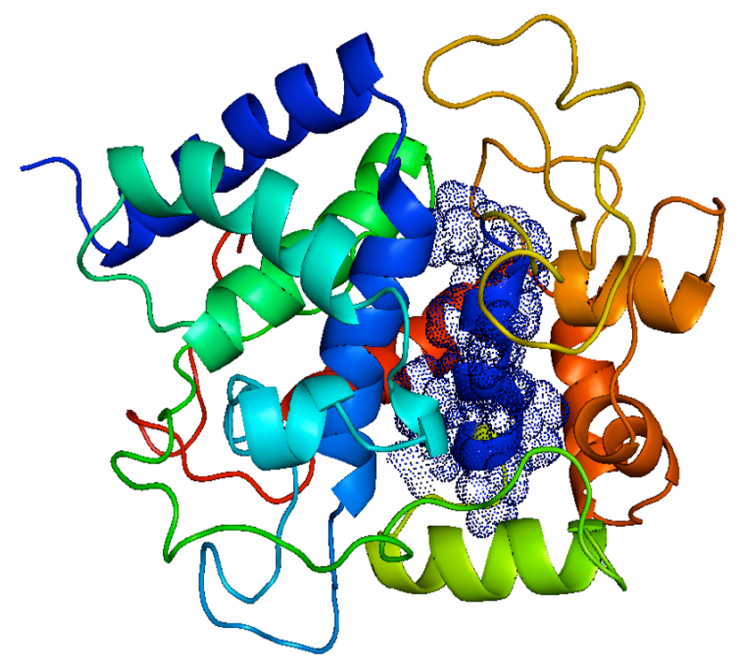

Figure 2: The final 3D model structure of SlAPX6 protein. This was obtained by Modeller 9v5 and verified employing PROCHECK, Verify3D and ProSA servers

\section{Homology modeling}

The 3D structure model of tomato SIAPX6 was generated by Modeller 9.16 [8]. Five models were generated with the lowest DOPE (Discrete Optimized Protein Energy) score was considered for further refinement and validation. Simultaneously another model was also generated by online web server employing Swiss model using same template [9]. GROMOS96 implemented incorporated in Swiss PDB Viewer The energy minimization was carried out employing

[10] Protein models and active site outputs were viewed by PyMOL molecular visualization tool.

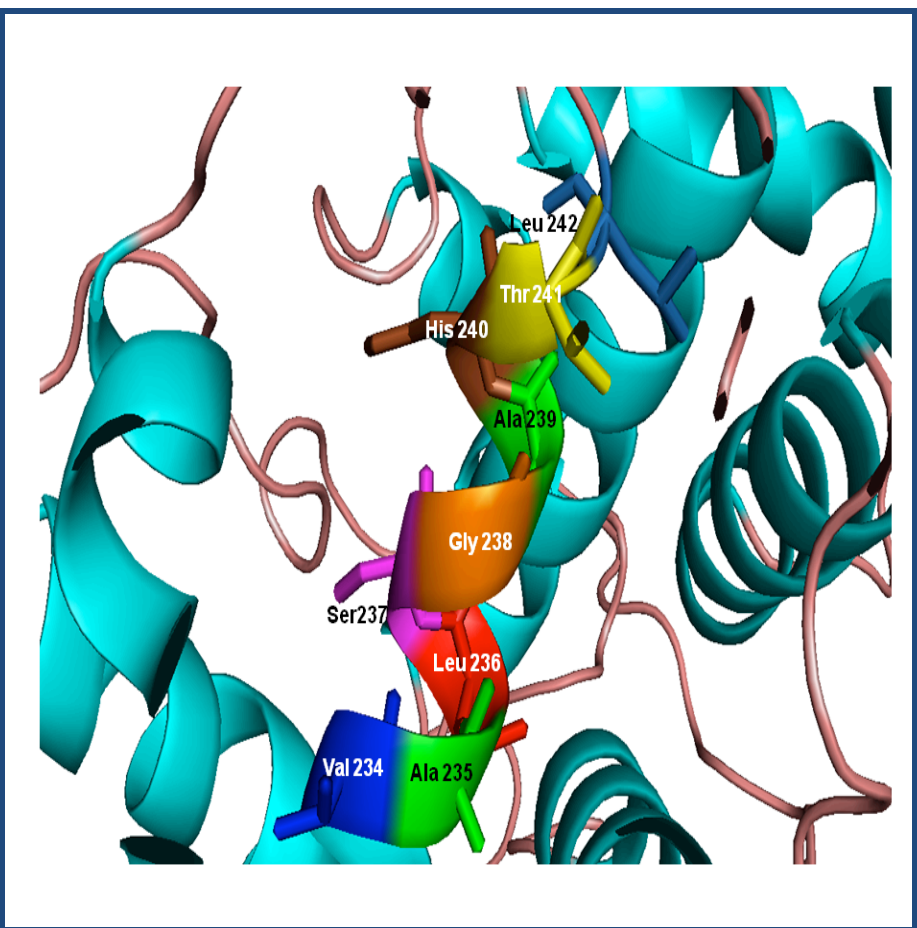

Figure 3 Active site residue prediction in SIAPX6 using discovery studio, active site mark with different colors val234 blue, ala235 green, leu236 red, Ser237 magenta, gly238 orange, ala239 green, his240 brown, thr241 yellow and leu242 skyblue.

\section{Validation of model}

The model was validated on the basis of stereochemically and geometrically constraints employing PROCHECK [11], ProSAWeb [12] and Verify 3d [13]. Root Mean Square Deviation (RMSD) investigation of the predicted structural model from its template was calculated by SUPERPOSE [14].

\section{Structural sequences alignment}

Structural sequences alignment of tomato SlAPX6 (Solyc11g018550.2.1) proteins and along with other PDB such as 1IYN, 2VCF, 2XIF, 2Y6B and template (PsAPX) was done using structure prediction of tomato SlAPX6 using PROMALS3D (http:// prodata.swmed.edu/promals3d/promals3d.php) was performed [15].

\section{Result \& Discussion:}

The length of SlAPX6 protein sequences is 421 amino acid residues long. Sequence search using BLAST identified the crystal structure with PDB ID: 1APX. The Pisum sativum SlAPX6 (PDB ID: 1APX) showed 45\% sequence identity to the query sequence with an E-value of 1e-59. ScanProsite server predicted the fragment VALSGAHT from 234- 242 residues as a motif (Figure 1). The sequence alignment revealed that the substrate binding interacting residues valine, alanine, leucine, serine, glycine alanine histidine and threonine were conserved both in SIAPX6 as target and PSAPX as template (Figure 2). These highly conserved residues might be functional in plant stress defense during fruit ripening and development in tomato (Figure 3). 

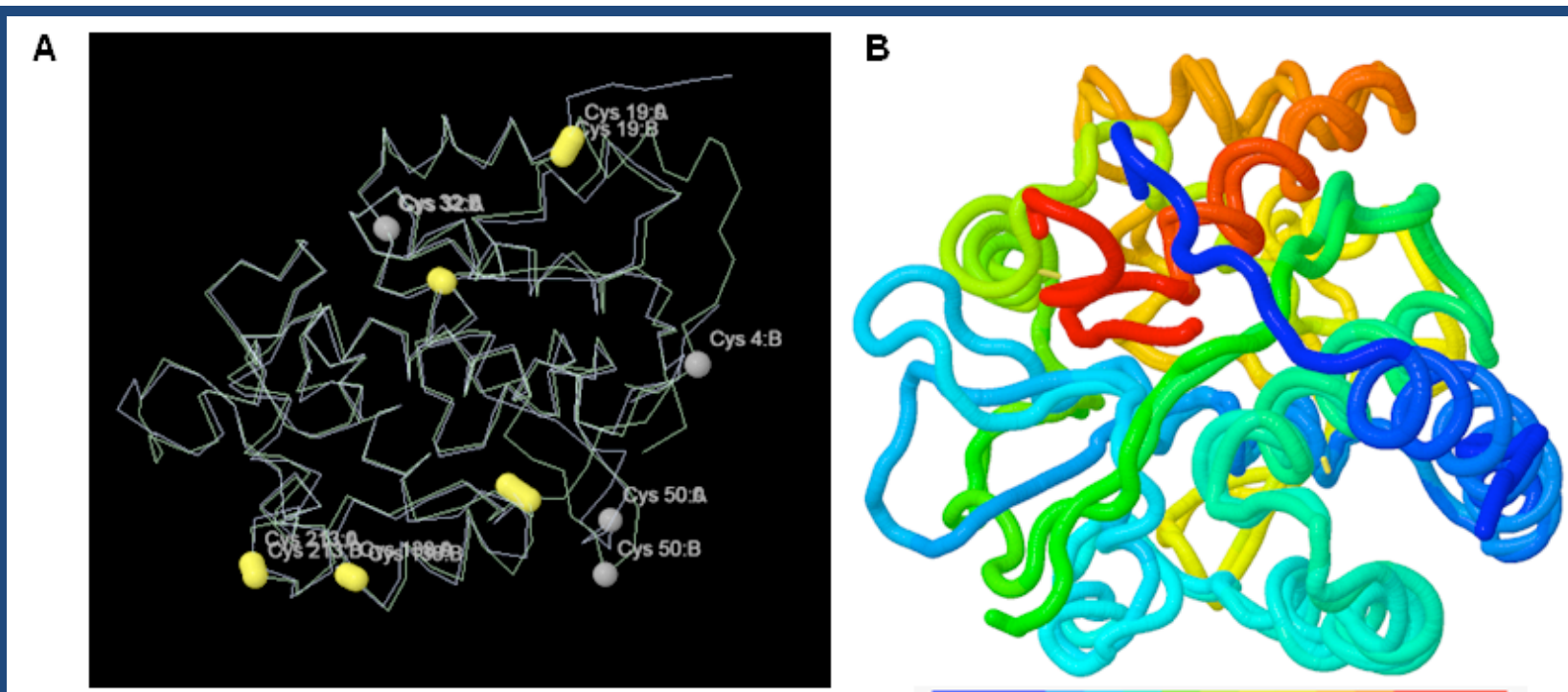

$\mathbf{N}, \mathbf{5}^{+}$

Figure 4: A) Superpose of model with disulphide linkage in protein chain; B) N- C Rainbow represent the protein chain with amino $\mathrm{N}$ terminal of protein are blue and carboxy $\mathrm{C}$ terminal are red.

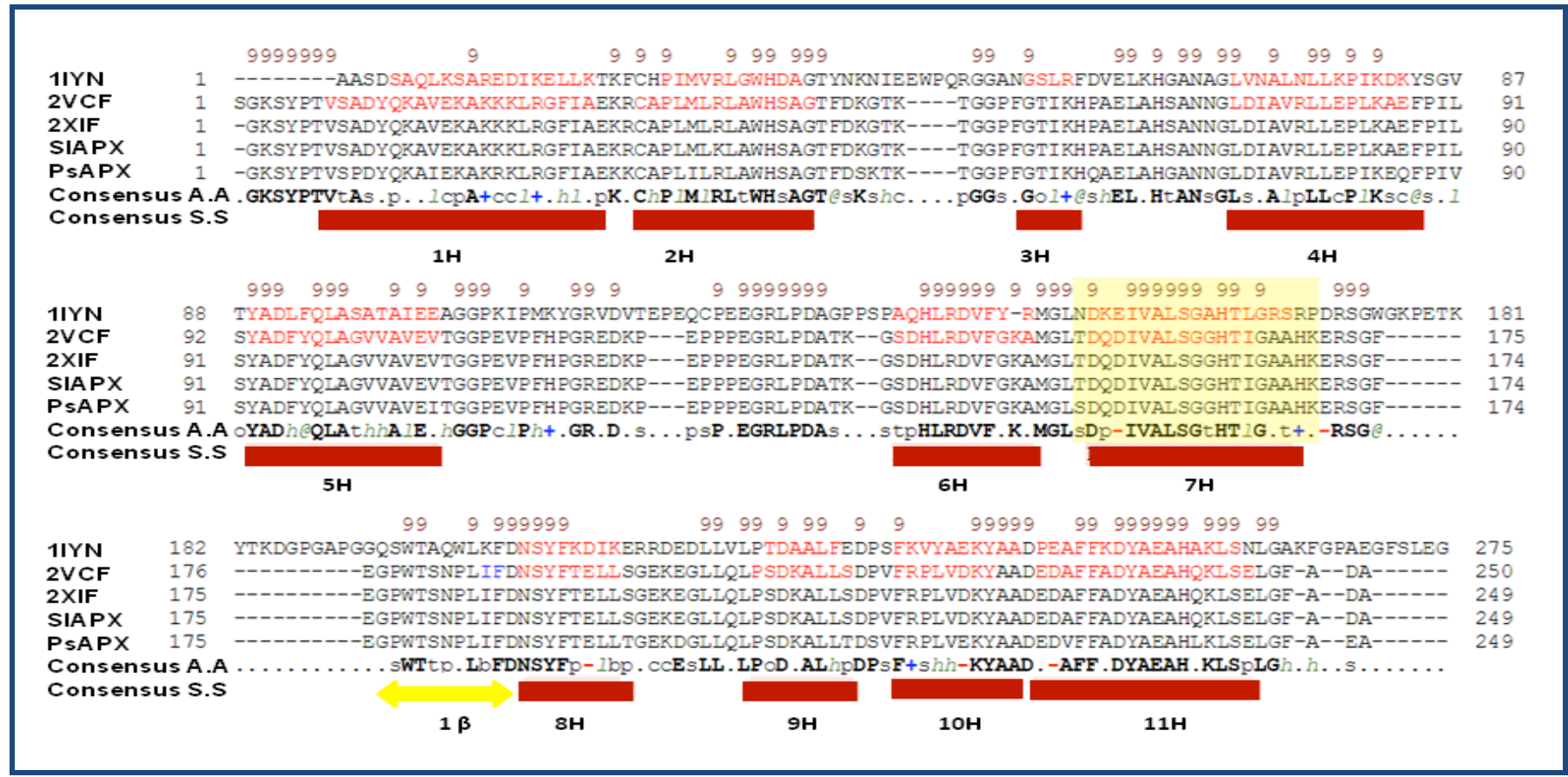

Figure 5 Structural sequences alignment of chloroplastic tomato SlAPX6 with its various homologous PDB such as 1IYN, 2VCF, 2XIF, 2Y6B and template (PSAPX) revealed that 11 alpha helices and 1 beeta strands were conserved in all the selected PDB id such as 1IYN, 2VCF, 2XIF, 2Y6B and template (PSAPX). This was generated using the PROMALS3D online web tools. The secondary structure elements were marked in colors red (helices) and yellow (beta sheet).

After that we have buildup structure for tomato SlAPX6 employing MODELLERTM. Five models were generated with the lowest Discrete Optimized Protein Energy, a statistical potential used to determine homology SIAPX6 models score of -24028.06 was considered to be thermodynamically stable and chosen for further refinement and validation. The stereochemically quality and accuracy of the predicted SIAPX6 model was evaluated by Procheck tool through Ramachandran plot. The MODELLERTM created model produced $94.6 \%$ residue found in most favored region, $4.9 \%$ residues in additionally allowed region, $0.5 \%$ residues in generously allowed and with no residues found in the disallowed region 
following the Ramachandran plot. This model is also compared with the Swiss Model with $95.0 \%$ residues in most favored region, $4.3 \%$ residues in additionally allowed region and $1.7 \%$ residues in generously allowed region. Consequently the model structure produced employing MODELLERTM was used for further analysis.

To further analyze the overall model quality target (SlAPX6) and template $(P S A P X)$ for compare check by ProSA web analysis server revealed a $Z$ score value measure of model quality total energy of the both structures such as template and target respectively of -8.78 of PSAPX and -8.32 of SlAPX6, Zscore analysis revealed a very good accuracy of our SIAPX6 model. Verify3D use for the determines the compatibility of an atomic model (3D) with its own residues by assigning a structural class based on its alpha, beta, loop, polar, non-polar. Therefore its comparing the results to good structures revealed $98.91 \%$ of the residues had a score $>=0.2$ for a best quality of our SIAPX6 model. The degree of structure similarity is measured using root-mean-square distance (RMSD) between corresponding atom pairs. RMSD examination of the tomato SIAPX6 model was measured from its template employing tools web server SuperPose (Figure 4). The RMSD between alpha carbon $(\mathrm{C} \alpha)$ and backbone deviation of Target (SlAPX6) and the template $\left(P_{S} A P X\right)$ crystal structure were $1.06 \AA$, and $1.08 \AA$ respectively. Thus, the MODELLERTM developed model was evaluated using several methods for reliability and precision.

Structural sequences alignment of chloroplastic tomato SlAPX6 with its various homologous PDB such as 1YN, 2VCF, 2XIF, Y6B and template $(P S A P X)$ revealed that 11 alpha helices and 1 beeta strands were conserved in all the selected PDB id such as $1 Y N, 2 V C F, 2 X I F, Y 6 B$ and template (PSAPX) (Figure 5). It illustrated high sequence likeness at the $\mathrm{N}$ terminal to $\mathrm{C}$ terminal with high helical region in whole protein sequences 11 helix and only one $\beta$ sheets. Thus, the model provides molecular insight to metal binding molecular function towards the understanding of photooxidative stress during flower to fruit development and ripping stage in tomato. The predicted model could be further explored for identification of substrate ligand binding sites, site directed mutagenesis and alter for specific role of functional site residues during catalysis.

\section{Conclusion:}

The thylakoid membrane bound tomato ascorbate peroxidase (SIAPX6) plays a major role in flower and fruit development in fleshy fruit during tomato ripening stage green, breaker and better protein engineering which may be useful to understand

red against photo-oxidative stress. Therefore, it is of interest to deduce its molecular function and propose mechanism of action. Thus, a homology structural model of the protein was constructed and analyzed further to infer molecular function. The model data in addition to other relevant post model analysis data proposes to provide molecular insight to heme binding molecular function towards the understanding of photo-oxidative stress tolerant in fleshy fruit tomato. Data highlights the importance of the heme-binding site in scavenging ROS from flesh fruit tomato during ripening.

\section{Acknowledgement:}

The authors thankfully acknowledge the financial support for DBT Biocare project, Government of India, New Delhi. The authors are grateful to the Nutritional Improvement of Crops Group, ICGEB, New Delhi, India for providing the necessary facilities, constant support and encouragement throughout the study.

\section{References:}

[1] Pandey S et al. Biochemistry Physiology 2015 4: 2

[2] Pandey $S$ et al. Bioengineering E Biomedical Science 2011 1:2

[3] Maruta T et al. Plant and Cell Physiol. 2010 51: 190

[4] Altshul SF et al. Nucleic Acids Res. 1997 25: 3389 [PMID: 9254694]

[5] http://www.rcsb.org/

[6] Thompson JD et al. Nucleic Acids Res. 1994 22: 4673 [PMID: 7984417]

[7] Gattiker A et al. Appl Bioinformatics. 2002 1: 107 [PMID: 15130850]

[8] Sali TL \& Blundell TL. J Mol Biol. 1993 234: 779 [PMID: 8254673]

[9] Schwede T et al. Nucleic Acids Res. 2003 31: 3381 [PMID: 12824332]

[10] Guex N \& Peitsch MC. Electrophoresis 1997 18: 2714 [PMID: 9504803]

[11] Ramachandran GN et al. J Mol Biol. 1963 7: 95 [PMID: 13990617]

[12] Wiederstein M \& Sippl MJ, Nucleic Acids Res. 2007 35: W407 [PMID: 17517781]

[13] Eisenberg D et al. Methods Enzymol. 1997 277: 396 [PMID: 9379925]

[14] Maiti R et al. Nucleic Acids Res. 2004 32: W590 [PMID: 15215457]

[15] Pei J \& Grishin NV. Bioinformatics 2008 23: 802 [PMID: 17267437]

Edited by $P$ Kangueane

Citation: Tripathi et al. Bioinformation 12(2): 44-47 (2016) License statement: This is an Open Access article which permits unrestricted use, distribution, and reproduction in any medium, provided the original work is properly credited. This is distributed under the terms of the Creative Commons Attribution License

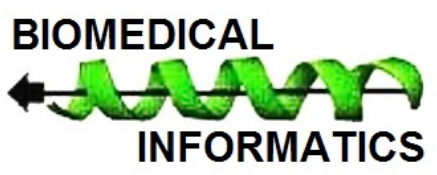

